\title{
Clinical Use of a Computer Assisted Reverse Total Shoulder Arthroplasty System: An Analysis of 1702 Cases
}

\author{
Alexander Greene ${ }^{1}$, Sandrine Polakovic ${ }^{1}$, Christopher Roche ${ }^{1}$, Yifei Dai ${ }^{1}$ \\ ${ }^{1}$ Exactech, Inc., Gainesville, FL, USA \\ alex.greenedexac.com
}

Disclosures: Alexander Greene (Exactech); Sandrine Polakovic (Exactech); Christopher Roche

(Exactech); Yifei Dai (Exactech)

\begin{abstract}
Placement of the glenoid component in reverse total shoulder arthroplasty (rTSA) is of paramount importance and can affect a patient's range of motion postoperatively. Preoperative planning and computer assisted surgery (CAS) can improve upon glenoid placement, but such systems for rTSA have experienced limited commercial success. Postoperative surgical reports from the first 1702 clinical cases of a commercially available CAS rTSA system were collected and analyzed for implant selection, implant placement, and incision start to incision close operative time, and compared to similar date cohorts for non-navigated cases. Navigated rTSA cases had a significantly longer incision time than non-navigated cases. Augmented glenoid components were used in a much higher percentage of navigated cases than non-navigated cases, suggesting that augmented glenoid components provide utility for correcting pathologic glenoid wear. The average resultant version and inclination of the implanted component increased with the size of augment used, suggesting there may not be a clear consensus on optimal version or inclination. Long term clinical follow up will need to be collected to determine if preoperative planning combined with more precise and accurate glenoid component positioning leads to improved clinical outcomes and implant longevity.
\end{abstract}

\section{Introduction}

Reverse total shoulder arthroplasty (rTSA) can be a challenging procedure. With limited access to bony landmarks of the scapula and no clear indicators of the mechanical axes for alignment, precise determination of glenoid version and inclination can be very difficult ${ }^{1}$. Numerous studies have demonstrated that malposition of the glenoid component leads to increased stresses in the glenoid 
Clinical Use of a Computer Assisted Reverse Total Shoulder Arthroplasty System: ... A. Greene et al.

bone as well as increased micromotion in the implant which may contribute to early glenoid loosening ${ }^{2}$. Using conventional free hand instrumentation, Iannotti et al. demonstrated that when surgeons believe they are aiming for neutral version/inclination pin placement in the glenoid, the angular accuracy in fact exceeds $\pm 10^{\circ}$ in both planes ${ }^{1}$.

3D preoperative planning alone has been shown to improve accuracy of glenoid implant placement ${ }^{1}$. To execute such a preoperative plan, patient specific instrumentation may improve accuracy, but the degree of improvement has recently come into question ${ }^{3}$. Computer assisted surgery (CAS) can improve upon glenoid placement, but such systems for rTSA have experienced limited commercial success. The purpose of this study is to examine the utility of a commercially available CAS rTSA system in 1702 clinical cases.

\section{Methods}

A CT based preoperative planning software and intraoperative rTSA navigation system was developed and launched in September of 2016. For the procedure, the surgeon templates the case with a preoperative $\mathrm{CT}$ scan by selecting and adjusting the glenoid component with respect to the scapula in $1 \mathrm{~mm} / 1^{\circ}$ increments and optimizing for factors such as implant coverage, vault penetration, and residual version/inclination correction (Figure 1). For rTSA glenoid implants, this system offers a standard nonaugmented component, a non-augmented $10 \mathrm{~mm}$ extended cage component, an $8^{\circ}$ posterior augmented wedge component, a $10^{\circ}$ superior augmented wedge component, and a combined $10^{\circ} / 8^{\circ}$ superior/posterior augmented wedge component. Once satisfied with the plan, the surgeon transfers the plan to the navigation unit.

During the procedure, the surgeon fixates a tracker to the coracoid and acquires points with a probe to match the preop CT to the bone. Preparation of the glenoid bone for implantation of the glenoid implant including drilling, reaming, and screw placement are all guided by the navigation system. A floating target and axis for the instrument is displayed and overlaid with the target for the preoperative plan on a 3D rendering of the scapula (Figure 2).

Since the launch of the system, over 4200 cases have been performed in 9 countries worldwide. Previous reported accuracy of the system allowed surgeons to place a glenoid component within $1.9^{\circ}$ of version, $2.4^{\circ}$ of inclination, $.7 \mathrm{~mm}$ of anterior-posterior (AP) placement, $1.1 \mathrm{~mm}$ of superior-inferior (SI) placement, and $1.1 \mathrm{~mm}$ of reaming depth with respect to their preoperative plans. Intraoperatively, the navigation system captures data on instrument position, implant selection, and implant placement. Postoperative surgical reports from the first 1702 rTSA clinical cases were collected and analyzed for implant selection, implant placement, and incision start to incision close operative time, and compared to similar date cohorts for non-navigated cases.

\section{Results}

For operative time, navigated rTSA cases lasted significantly longer than non-navigated cases ( $p<$ .05 ), when compared to a similar date range cohort (Nav: $93 \pm 31$ minutes; Non-nav: $71 \pm 28$ minutes).

For implant usage in the navigated cohort, standard glenoid components were used $28.7 \%$ of the time, compared to total sales data for the same date range of $80.5 \% .8^{\circ}$ posterior augmented glenoid components were used $35.5 \%$ of the time, compared to total sales data of $9.2 \% .10^{\circ}$ superior augmented 
Clinical Use of a Computer Assisted Reverse Total Shoulder Arthroplasty System: ... A. Greene et al.

glenoid components were used $14.7 \%$ of the time, compared to total sales data of $4.5 \% \cdot 10^{\circ} / 8^{\circ}$ superior/posterior augmented glenoid components were used $18.9 \%$ of the time, compared to total sales data of $3.9 \%$. Lastly, extended cage glenoid components were used $2.1 \%$ of the time, compared to total sales data of $2.0 \%$.

For resulting version and inclination, standard non-augmented glenoid components were implanted in $1.4 \pm 3.0^{\circ}$ of retroversion and $-0.5 \pm 1.4^{\circ}$ of inclination, extended cage non-augmented glenoid components were implanted in $1.0 \pm 3.9^{\circ}$ of anteversion and $-1.4 \pm 3.7^{\circ}$ of inclination, $8^{\circ}$ posterior augmented glenoid components were implanted in $3.1 \pm 3.2^{\circ}$ of retroversion and $-0.1 \pm 1.2^{\circ}$ of inclination, $10^{\circ}$ superior augmented glenoid components were implanted in $3.4 \pm 4.2^{\circ}$ of retroversion and $0.1 \pm 1.1^{\circ}$ of inclination, and $10^{\circ} / 8^{\circ}$ superior/posterior augmented glenoid components were implanted in $2.1 \pm 3.1^{\circ}$ of retroversion and $-0.6 \pm 2.2^{\circ}$ of inclination.

\section{Discussion}

Navigated cases took significantly longer than non-navigated cases. Anecdotal individual surgeon time data suggests a learning curve, where the surgeon takes longer at first to get used to the system before reducing his or her total incision time after a number of cases. This observation is also seen in navigated arthroplasty procedures in other joints ${ }^{4}$.

A usage rate of $69.2 \%$ augmented glenoid components in the navigated cohort suggests the utility of augmented components in correcting pathologic glenoid wear. The observation of a much higher percentage of augmented glenoid components in the navigated cohort vs. the non-navigated cohort may be the result of the surgeon being able to more accurately visualize pathologic wear patterns on 3D models vs. 2D CT or plain films, which lends surgeons to use augmented components more frequently to correct this wear ${ }^{5}$. This high frequency of augmented glenoid component use and inconsistent degree of version correction in each implant category paired with high standard deviations raises questions about how much retroversion for a rTSA is optimal. This trend was not observed for inclination, where surgeons planned for $0^{\circ}$ or less of inclination. This is likely due to surgeons' desire to inferiorly tilt the implant to avoid scapular notching and inferior impingement.

\section{Significance/Clinical Relevance}

This navigation system may represent an improvement in implant placement and potentially implant longevity. Further study is required to see if these improvements in preoperative planning and more accurate and precise glenoid component positioning are associated with better clinical outcomes in the short and long term. Long term clinical follow up is being collected on both navigated and nonnavigated patient cohorts and will be reported at a future date.

\section{References}

1. Iannotti J, Baker J, Rodriguez E, Brems J, Ricchetti E, Mesiha M, et al. Three-Dimensional Preoperative Planning Software and a Novel Information Transfer Technology Improve Glenoid Component Positioning. J Bone Jt Surg. 2014 May 7;96(9):e71. 
Clinical Use of a Computer Assisted Reverse Total Shoulder Arthroplasty System: ... A. Greene et al.

2. Farron A, Terrier A, Buchler P. Risks of loosening of a prosthetic glenoid implanted in retroversion. J Shoulder Elbow Surg. 2006 Jul;15(4):521-6.

3. Lau SC, Keith PPA. Patient-specific instrumentation for total shoulder arthroplasty: not as accurate as it would seem. J Shoulder Elbow Surg. 2018;27(1):90-95.

4. Smith BR, Deakin AH, Baines J, Picard F. Computer navigated total knee arthroplasty: the learning curve. Comput Aided Surg. 2010;15(1-3):40-8.

5. Amini MH, Ricchetti ET, Iannotti JP. Three-Dimensional Templating and Use of Standard Instrumentation in Primary Anatomic Total Shoulder Arthroplasty. JBJS Essent Surg Tech. 2017;7(3):e28. Published 2017 Sep 27. doi:10.2106/JBJS.ST.17.00009 


\section{Figures}
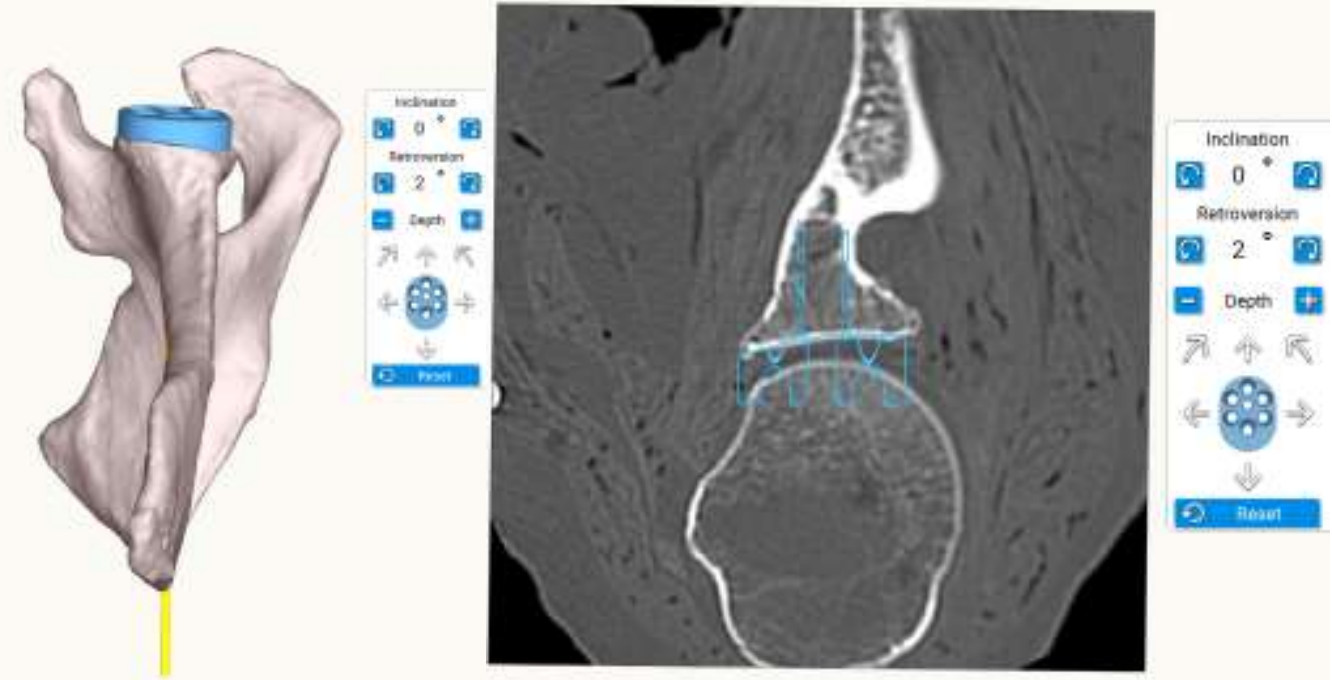

Figure 1: Preoperative Planning Software User Interface

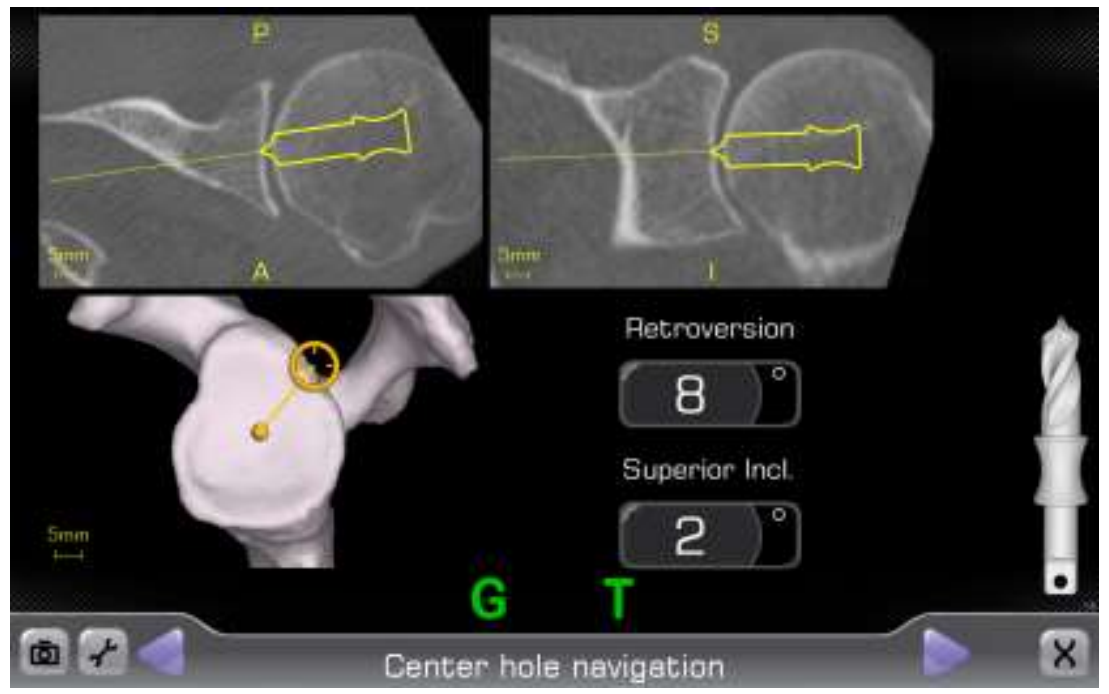

Figure 2: Navigation Software User Interface 